\title{
Acute mental health nurses as therapists: A survey of the opinions of trainee nurse therapists and their patients in acute mental health provision
}

\author{
Billy Mathers \\ School of Health, University of the West of Scotland, Paisley, Scotland
}

Correspondence: Billy Mathers. Address: School of Health, University of the West of Scotland, High Street, Paisley PA1 2BE, Scotland. Email: billy.mathers@uws.ac.uk.

Received: October 31, 2012

DOI : 10.5430/jnep.v3n10p50
Accepted: January 16, $2013 \quad$ Online Published: March 21, 2013

URL: http://dx.doi.org/10.5430/jnep.v3n10p50

\section{Abstract}

Background/Objectives: The use of psychosocial interventions has been shown to improve the functioning of individuals with serious mental disorders such as schizophrenia. Such skills have been taught to mental health nurses in community settings for many years and have been well evaluated but there is a paucity of evidence for their use on acute inpatient wards. This study evaluates two teaching modules convened for qualified mental health nurses who are working in acute adult inpatient wards in several mental health units. The purpose of the modules is to teach trainees psychosocial interventions to equip them to care for patients with severe mental illness. The study's objectives are to evaluate the effectiveness of the teaching on the modules and also to evaluate the trainees' success in transferring the interventions which they learned into clinical practice.

Methods: A quasi experimental design was adopted to ascertain whether or not trainees' perceptions of their practice had changed 'post module' and to investigate whether trainees' patients reported differences in their nursing care after training. A questionnaire was administered to each trainee (experimental group) before and after the modules to elicit their opinion of their ability in caring for patients with severe mental health problems. Their responses 'pre module' were then compared to their responses 'post module'. The effectiveness of the modules was further evaluated by comparing the experimental group's 'post module' responses to those of a group matched for length of service and experience who had not undertaken the modules (reference group). To measure the trainees' transfer of psychosocial interventions into practice, a questionnaire was administered to the patients for whom they acted as 'named nurse' throughout their stay on the ward. Their responses were compared to patients for whom the reference group acted as 'named nurse'. The scales were tested for reliability using the Cronbach alpha test and statistical significant was assessed using both paired $t$-test and Wilcoxon's.

Results: The study found that the modules were effective in teaching trainees psychosocial interventions, but that they found implementation difficult.

Conclusions: The modules were effective in giving trainees more confidence in caring for patients on acute wards. However, ways need to be found to empower trainees to transfer the skills which they have learned in the modules into clinical practice.

\section{Key words}

Psychosocial interventions, Acute mental health nurses, Severe mental illness 


\section{I ntroduction}

Traditional treatment for severe mental illness (SMI) such as schizophrenia has been chiefly antipsychotic medication but more recently the use of psychosocial interventions (PSI) have been shown to reduce the disability associated with these disorders. As one review ${ }^{[1]}$ states (p2) "psychoeducational interventions significantly decreased relapse or re-admission rates at nine to 18 months follow-up compared to standard care.” PSI skills such as the psychoeducation mentioned above have been used by mental health nurses in community settings for many years and have been well evaluated ${ }^{[2]}$ but the evidence is relatively sparse for acute inpatient wards. Implementing PSI alongside medication in inpatient settings could improve the patient's quality of life here too, and perhaps reduce length of stay ${ }^{[3]}$. Given the pressure on acute beds, mental health service providers welcome interventions that can result in a reduction in length of stay. Other theorists imply that such training should be universal in order to promote best practice ${ }^{[4]}$.

Reports over the past decade and beyond have indicated that higher numbers of patients with SMI were being admitted to acute inpatient wards and recommended further training for staff to equip them to work with a client population with more complex needs ${ }^{[5,6]}$. In response to these developments, the mental health team at the university where the research reported here was conducted decided to offer a course of training in PSI for health professionals in acute mental health settings. Whilst there had been a similar course for community workers in the university for some years (Thorn course), there was no such course for inpatient workers and as a result the modules were developed.

The precise nature of PSI is difficult to pinpoint as there is considerable variation in description and practice. However, in addition to psychoeducation mentioned above, a wide range of components have been included. These include patient engagement, comprehensive assessment, cognitive-behavioural skills (e.g. psychological treatment of persistent symptoms), early intervention in psychosis, family interventions and medicines management ${ }^{[7-10]}$. All of the above components were included in the PSI modules which were offered.

PSI and similar skills have been taught to mental health nurses in the community for some time ${ }^{[2,10]}$. Although such studies were well evaluated, there is less evidence of favourable outcomes on acute inpatient wards. PSI training for nursing staff on inpatient wards was conducted as outlined in two studies ${ }^{[11,12]}$. Possible problems identified with such training was that the short term of hospitalisation in acute wards might make it difficult for nursing staff to work intensively with patients due to lack of time. They also highlight limited management support for staff to continue interventions after the training has ceased, a theme also identified by another study ${ }^{[13]}$ which outlined similar ward based PSI training.

The above studies unite in their evaluation that acute inpatient services have difficulty in terms of providing therapeutic involvement by nursing staff. These observations provided the impetus for the development of the PSI modules being evaluated in this study. This paper is an evaluation of the effectiveness of psychosocial interventions (PSI) training modules offered to acute nursing staff (trainees) and the trainees' subsequent attempts to apply their new skills in clinical practice on acute inpatient wards.

\section{Methods}

\subsection{Setting and design}

A quantitative, quasi experimental design was adopted to ascertain whether or not trainees' perceptions of their practice changed 'post module'. Randomisation is absent from the present study because all trainees volunteered for the modules and all who applied were offered a place. 


\subsection{Evaluation of the modules}

The experimental group comprised of trainees from the PSI modules $(n=20)$ and were recruited at the training modules. After a verbal introduction, letters were sent to the trainees inviting them to participate and they were only included in the study after a signed agreement had been received. All trainees agreed to participate.

The reference group ( $n=20$ ) were matched for length of experience and were typical of nurses in these settings who had not received PSI training. Members of the reference group were suggested by the trainees and were colleagues who worked either on the same ward or an adjoining ward. After an introduction by the trainees, they were approached to take part in the study. The mean length of experience for the experimental group was five years while the mean length of experience for the reference group was 5 years 6 months. The mean age of the experimental group was 31 and the mean age of the reference group was 33. The experimental group was comprised of 12 female staff and the reference group 13 female staff. None of the reference group had been exposed to formal PSI training.

Trainees' perception of their training was measured using a questionnaire devised for the study known as the 'Trainees Assessment of Post-registration Skills' (TAPS). The 33 items in the questionnaire were developed to elicit information about the pre and post registration training which trainees had received. Trainees completed the TAPS before the modules began 'pre test' and then on the final day of the modules after the teaching was completed, 'post test' as part of the evaluation of the modules. Nurses in the reference group completed the TAPS on one occasion.

Since the study was primarily an evaluation of the PSI modules, the 31 quantitative items chosen for the questionnaire covered issues of assessment and intervention concerning the types of mental disorder featured in the modules. Including both assessment and interventions in the questionnaire was important in order to reflect the teaching, as one module focussed upon assessment and the other upon interventions. The teaching on the modules included the assessment, intervention and evaluation of depression self-harming and suicide intent. Also covered were sessions on working with patients with generic issues such as anxiety states, personality disorder, ethnic issues, drug induced psychosis, schizophrenia, bipolar disorder, risk assessment and forensic issues. Therefore, trainees were asked how they felt their pre and post training had equipped them to provide assessment and intervention for these patients. Trainees were asked to indicate on a five point Likert scale how they perceived their ability to care for patients with these disorders.

The PSI issues which had been covered in the modules were: engagement, evidenced based assessment (using KGV, LUNSERS and SFS), brief interventions with psychosis, medication management, the stress vulnerability model and family interventions. PSI is designed for work with SMI like hallucinations and delusions and these topics were included in the questionnaire. For example item 21 stated "my pre-registration MH nurse training \& the post reg. courses/modules I have taken have equipped me with the intervention skills to reduce patients' hallucinations using recognised psychosocial interventions.” In this way evaluation of the PSI component of the modules was covered. The questionnaire was piloted by gaining opinions from trainees on the previous modules who were not included in the present study.

\subsection{Evaluation of trainees' application of the skills in practice}

A quantitative, quasi experimental design was adopted to ascertain whether or not trainees were applying their newly acquired skills in clinical practice. This was achieved by administering a questionnaire to patients to ascertain how they had been nursed by the trainees. Patients were recruited through their named nurses. The questionnaire was administered by the named nurse in order to add objectivity to the process, although the researcher was available to answer any questions which might arise. The questionnaire took 30 minutes to complete on average. Ethical clearance for this study was obtained from an ethical committee appointed by the local health authority.

A 21 item questionnaire was administered to 30 'primary patients' of the experimental group and 30 'primary patients' of the reference group. This was to ascertain whether the trainees spent more time in assessment and intervention than the reference group. This was known as 'Patients’ Assessment of Trainees' Skills’ (PATS). 
Once again, as in the trainees' questionnaire, the focus of the questions was around the subject matter of the modulesSMI-and how much time had been spent by the primary nurse in talking about issues related to this. The idea was to compare responses of the trainee (experimental) group's patients and the responses of the reference group patients to see if the modules had changed the trainees' practice in any way. A pilot study for the PATS was undertaken with the help of patients who were not involved in the research.

The items in the PATS questionnaire were chosen to gain an indication of whether or not the PSI skills which were taught to trainees were being used in practice. The items were based on the teaching the trainees had received in the modules (see Tables 1 and 2). These questions were initially asked in relation to when the patients were first admitted to assess trainees' use of assessment skills in PSI. The same questions were then asked in relation to a later period, when the patients had been settled on the ward. This was to assess trainees' interventions in PSI. The same questionnaire was administered to the patients of the reference group in order to ascertain the differences in responses.

\section{Results}

\subsection{Trainee questionnaire}

All 20 trainees who completed the modules returned both the pre and post module TAPS questionnaires. A five point Likert scale (strongly agree to strongly disagree) was used to elicit the data. The scale was tested for reliability using the Cronbach alpha test. One researcher ${ }^{[14]}$ states that a value for Cronbach's alpha coefficient of more than .7 is an indication of good reliability. In the current study, the Cronbach alpha coefficient was .95, so the scale can be considered internally consistent within the sample.

The statement trainees were responding to was; "my pre-registration MH nurse training \& the post reg. courses/modules I have taken have equipped me with the skills to work adequately with patients in .........". With the exception of two items (27 and 28, interventions and evaluation in suicidal ideation) trainees had higher mean responses after the modules than before, suggesting that they felt better equipped to address the range of clinical skills outlined in the questionnaire after attending the modules.

Whether or not trainees' greater confidence 'post-modules' was statistically significant, was assessed using both paired $t$-test and Wilcoxon's. The Wilcoxon's test was used as a precaution because, although the distributions were normal, the Likert scale is ordinal. However, significance levels were the same using both these tests and only the results of the t-test are reported. Although some researchers question the use of the t-test with Likert data, one study ${ }^{[15]}$ compared the "t" test with the Mann-Whitney-Wilcoxon for five-point Likert items and their conclusion was that for five-point Likert items, the $t$ test and the Mann-Whitney-Wilcoxon generally have similar power.

It has been stated ${ }^{[14]}$ that if the probability value is less than .05 then it can be concluded that there is a statistically significant difference between the two scores. However, given the number of analyses being conducted, significant differences at the .05 and .01 levels should be interpreted with caution.

Trainees felt significantly more confident of their skills after attending the modules on 12 out of the 31 items, or, using the more cautious .001 level of significance, on six items. Items associated with significant improvement related to assessment and intervention in psychosis, delusions and hallucinations, the particular focus of the modules. Before statistically testing the difference between trainees' pre and post module total scale scores, the normality of the scale's distribution was examined. As the distributions were reasonably normal, a paired-samples $t$ - test was conducted to evaluate the impact of the intervention (training) on trainees' overall scores. 
There was a statistically significant difference in scores from pre module $(M=103.25, S D=20.20)$ to post module $(\mathrm{M}=120.6, \mathrm{SD}=13.69), \_t(19)=4.16, \ldots<.001$. The eta squared statistic of .47 indicates a large effect, according to one source ${ }^{[14]}$ who gives the following rule of thumb for interpreting eta squared: $.01=$ small effect, $.06=$ moderate effect, $.14=$ large effect. This indicates that trainees' perceptions were that their knowledge and confidence had improved substantially.

\subsection{Trainees' and reference group compared}

The same questionnaire was used to compare trainees' post module responses against a reference group of their peers. These were colleagues on acute settings with similar experience and training who had not undertaken the PSI or other similar modules. Differences between the pre module trainee response and those of the reference group were tested for statistical significance. The same significant group differences were identified by both tests and therefore only $t$-test are reported. At the item level there was only one statistically significant difference between the groups: evaluation of depression; $t(38)=2.2, p<.05$ and this may not be true because of the multiple comparisons. The trainees were on average less confident than the reference group. There was no group difference in the total scores. This would indicate that the reference and trainee groups were fairly equivalent before intervention.

Comparing trainees' post module responses with those of the reference group, at the item level, it was quite striking to see a significant difference in the items related to psychosis between the two groups, trainees rating these items substantially higher that the reference group. This indicates that the main focus of the teaching modules had achieved its purpose in helping trainees work with this client group. The post module trainees reported being significantly more confident than the reference group on 10 out of the 31 items. For no item did the reference group report significantly greater confidence than post module trainees.

Similarly, differences between the post module trainees total scale scores and those of the reference group were tested for statistical significance using an independent samples t-test. The trainees were significantly more confident than the reference group $\left(\mathrm{M}=120.6, \mathrm{SD}=13.69 \& \mathrm{M}=110.1, \mathrm{SD}=12.6\right.$ respectively; $\left.t(38)=2.514, p_{-}=.016\right)$. The eta squared of .14 indicates a large effect of the intervention between the groups using the above rule of thumb. This would seem to indicate that the modules had been effective in preparing the trainees to work in the specified area.

\subsection{Patient's questionnaire}

Table 1 shows the mean and standard deviations for items 1-10. The questionnaire made the statement 'when I first came on to ...... ward my nurse spent a lot of time asking about.....' referring to the initial assessment process.

Table 1. Comparison of trainee and reference group 'PATS' assessment scores

\begin{tabular}{llll}
\hline Item & $\begin{array}{l}\text { Trainee group } \\
\text { Mean (SD) }\end{array}$ & $\begin{array}{l}\text { Reference group } \\
\text { Mean (SD) }\end{array}$ & \multicolumn{1}{c}{ t value } \\
\hline $\begin{array}{l}\text { 1. 'Suspicious ideas which other people did not seem to have' } \\
\text { 2. 'Hearing voices in my head' }\end{array}$ & $3.3(1.3)$ & $2.6(1.3)$ & -2.09 \\
3. 'Any unusual beliefs which other people did not seem to have' & $3.03(1.2)$ & $3.23(1.3)$ & -.74 \\
4. 'About the side-effects of my medication' & $3.2(1.1)$ & $2.8(1.2)$ & -1.30 \\
5. 'If I had any plans to harm/injure/kill myself' & $3.7(1)$ & $3.27(1.3)$ & -1.34 \\
6. 'How any recreational drugs (eg cannabis) I had taken affects my & $3.2(1.2)$ & $2.9(1.3)$ & -1.41 \\
thinking' & $3.47(1)$ & $3.1(1.2)$ & -.95 \\
7 'How I got on with my family and friends' & $3.13(1)$ & $3(1.1)$ & -1.31 \\
8. 'My daily activities and my energy to do these activities' & $2.7(1.2)$ & $2.67(1.3)$ & -.48 \\
9. 'Periods when I feel over excited or too high (energetic)' & $3.0(1.2)$ & $2.8(1.4)$ & -.11 \\
10. 'Relationship break-up/ divorce/ redundancy/ bereavement' & & -.60 \\
\hline
\end{tabular}


The items (1-10) are related to the assessment process which was integral to the teaching of the modules. The first three items were particularly relevant to the modules as the main focus of the modules was working with SMI symptoms. Item 1 which roughly equates to the medical diagnosis 'paranoia' saw higher mean responses from the trainee group than the reference group. This trend continued in item 2 which roughly equates to 'auditory hallucinations' and item 3 (delusions). These findings indicate outcomes in the right direction for the evaluation of the modules. However, the difference between trainee and reference group patients was only statistically significant for Item 1.

Table 2. Comparison of trainee and reference group 'PATS' intervention scores

\begin{tabular}{|c|c|c|c|}
\hline Item & $\begin{array}{l}\text { Trainee group } \\
\text { Mean (SD) }\end{array}$ & $\begin{array}{l}\text { Reference group } \\
\text { Mean (SD) }\end{array}$ & $t$ value \\
\hline 11. 'Suspicious ideas which other people did not seem to have' & $2.83(1.2)$ & $2.8(1.2)$ & -.11 \\
\hline 12. 'Hearing voices in my head' & $3.27(1.1)$ & $2.73(1.3)$ & -1.74 \\
\hline 13. 'Any unusual beliefs which other people did not seem to have' & $2.67(.88)$ & $2.67(1.2)$ & .0 \\
\hline 14. 'Yy medication (including side-effects)' & $3.27(1.0)$ & $3.03(1.2)$ & -.82 \\
\hline 15. 'If I had any plans to harm/injure/kill myself' & $3.4(1.0)$ & $3.20(1.3)$ & -.66 \\
\hline 16. 'How any use of recreational was affecting my thinking' & $3(1.1)$ & $2.93(1.2)$ & -.23 \\
\hline 17 'How I got on with my family and friends’ & $3.47(1)$ & $3.17(1.05)$ & -1.15 \\
\hline 18. 'My daily activities and my energy to do these activities' & $3(1)$ & $3,23(1.0)$ & .91 \\
\hline 19. 'Periods when I feel over excited or too high (energetic)' & $2.97(1)$ & $2.80(1.1)$ & -.63 \\
\hline 20. 'Relationship break-up /divorce/ redundancy/ bereavement' & $2.97(1.1)$ & $2.73(1.26)$ & -765 \\
\hline 21. 'My nurse could have spent more time talking to me’' & $3.37(1.1)$ & $3.57(1.1)$ & .703 \\
\hline
\end{tabular}

In table 2, Items 11-20 made the statement 'during my time on ....ward my nurse met with me regularly to discuss....' referring to interventions carried out after the initial assessment period. This was to ascertain whether the trainees were using intervention skills taught on the modules. Each variable is itemized in the preceding table. These items are used as a measure of interventions which were also integral to the teaching of the modules. Therefore, it was expected that trainees would spend more time talking to patients in the 1-21 items. For example, items 2 and 12 'hearing voices in my head' were key areas for the experimental group (trainees) as this focused upon the assessment and management of patients' auditory hallucinations. Items 3 and 13 were also key areas for the trainees as they describe work with patients 'unusual beliefs' (delusional thinking) There were no statistically significant differences between the responses of the groups of patients. This indicates that 'primary' nurses in the trainee group, although carrying out slightly more extensive assessment for psychosis did not carry out more extensive interventions.

The implications which can be drawn from the above are that, overall, there are no marked differences between responses of trainees' patients and the patients in the reference group. This suggests that although trainees felt they had gained PSI skills to enable them to work with the SMI client group, they found these skills difficult to implement in practice.

\section{Discussion}

Generally speaking, trainees' scores showed greater confidence after undertaking the modules. The highest improvement was seen in the items that cover psychotic symptoms. There was found to be a statistically significant difference between the two scores. This reinforces the notion that trainees' attitudes had become more positive after completing the modules, precisely in those areas addressed by the modules. The difference in the trainees' post module scores compared to reference group scores also indicates that the modules had served their purpose.

A small increase in time that the trainees spent with patients in assessment and interventions in comparison with the reference group was found to be not statistically significant. The rationale for the patients' questionnaire was to ascertain the length of time patients felt that trainees were involved in a therapeutic role. It was to be expected that the trainees, who had been instructed in lengthy assessments and interventions would spend longer with patients than their colleagues. 
Although the questionnaire did not assess the quality of interaction, at least one report ${ }^{[6]}$ indicates that staff on acute wards were deemed by patients not to be spending enough time in interventions. Therefore, asking patients their perceptions of time of interventions of trainees with patients in comparison to reference group was considered important.

The problems of acute staff in establishing therapeutic engagement have been well documented in the introduction to this paper. In addition, two studies ${ }^{[16,17]}$ analyzed fieldwork diaries to document lessons which were learned from working on acute wards. It was suggested that blocks to therapeutic involvement were low levels of permanent staff, insufficient beds and anxiety about risk assessment and other containment issues.

\section{Conclusion}

This paper describes modules which taught PSI to mental health nurses working in acute inpatient wards. The modules appear to have been successful in giving trainees more confidence in working with SMI patients but there is little evidence that they were able to use the skills in practice. This paper has also outlined evidence from other studies which indicate reasons for lack of therapeutic involvement on acute wards, including lack of resources lack of leadership from managers and poor staffing levels.

Other research has suggested that trainees need to have PSI assessments integrated into PSI care plans and managers should be trained in PSI and committed to its use by their staff. Also, ongoing supervision in PSI needs to be provided by academic staff and trainees need to liaise more closely with consultants regarding patient discharge ${ }^{[3]}$. In order for acute inpatient mental health wards to achieve their full therapeutic potential these factors will need to be addressed so that skills learned on such modules can be utilised effectively in the future.

\section{References}

[1] Pekkala E, T, Merinder L.B. Psycho-education for schizophrenia. Cochrane Database of Systematic Reviews, 2, Art. No.: CD002831. http://dx.doi.org/10.1002/14651858.CD002831

[2] Brooker, C., Saul, C., Robinson, J., King, J. and Dudley, M. 'Is training in psychosocial interventions worthwhile? Report of a psychosocial intervention trainee follow-up study.’ International Journal of Nursing Studies. 2003; 40: 731-747. http://dx.doi.org/10.1016/S0020-7489(03)00013-0

[3] Mathers, B. Acute mental health nurses: comprehensive practitioners or specialist therapists? Journal of Psychiatric and Mental Health Nursing. 2012; 19: 47-52. PMid:22070390 http://dx.doi.org/10.1111/j.1365-2850.2011.01752.x

[4] Fleming, M., Savage-Grainge, A. P., Martin, C. R., Hill, C., Brown, S., Buckle, et al. 'The role of intrinsic factors in the implementation of psychosocial interventions into routine clinical practice.' Journal of Mental Health Training, Education and Practice. 2008; 3(2): 32-41. http://dx.doi.org/10.1108/17556228200800013

[5] Department of Health. 2002. Mental Health Policy Implementation Guide: Adult Acute Inpatient Care Provision. Department of Health: London

[6] Sainsbury Centre for Mental Health. 2006. The Search for Acute Solutions; improving the quality of care in acute psychiatric wards. SCMH, London.

[7] Bradshaw, T, Lovella, K, Richards, D. PSI and COPE, Mental Health Nursing. 2000; 20 (8): 10-14.

[8] McKeown, M., McCann, G. \& Forster, J. Adapting psychosocial interventions to inpatient environments. in Harris, N., Williams, S. \& Bradshaw, T. (eds) Psychosocial interventions for people with Schizophrenia. Macmillan: London 2003.

[9] Burns, T and Firn Assertive Outreach In Mental Health: A Manual For Practitioners. Oxford University Press: New York 2002

[10] Turkington, D. Kingdon, D. and Turner, T. 'Effectiveness of a brief cognitive-behavioural therapy intervention in the treatment of schizophrenia’. British Journal of Psychiatry. 2002; 180: 523-527. PMid:12042231 http://dx.doi.org/10.1192/bjp.180.6.523

[11] Baker, J.A. 'Developing psychosocial care for acute psychiatric wards'. Journal of Psychiatric \& Mental Health Nursing. 2000; 7(2): 95-100. http://dx.doi.org/10.1046/j.1365-2850.2000.00267.x

[12] Baker J.A., O'Higgins, H., Parkinson, J. and Tracey, N. 'The construction and implementation of a psychosocial interventions care pathway within a low secure environment: a pilot study’. Journal Psychiatric and Mental Health Nursing. 2002; 9(6): 737-739. http://dx.doi.org/10.1046/j.1365-2850.2002.00503.x 
[13] McCann, E. and Bowers, L. 'Training in cognitive behavioural interventions in acute psychiatric inpatients wards'. Journal of Psychiatric and Mental Health Nursing. 2005; 12: 215-222. PMid:15788040 http://dx.doi.org/10.1111/j.1365-2850.2004.00822.x

[14] Pallant, J. SPSS Survival Manual. Open University Press: Maidenhead. 2001

[15] de Winter, J. C. F. and Dodou D. Five-Point Likert Items: t test versus Mann-Whitney-Wilcoxon. Practical Assessment, Research \& Evaluation. 2010; 15(11): 1-16.

[16] Brennan, G., Flood, C. and Bowers, L. 'Constraints and blocks to change and improvement on acute psychiatric wards - lessons from the City Nurses project.’ Journal of Psychiatric and Mental Health Nursing. 2006; 13(5): 475-482. PMid:16965464 http://dx.doi.org/10.1111/j.1365-2850.2006.00956.x

[17] Flood, C., Brennan, G., Bowers, L., Hamilton , B., Lipang, M. and Oladapo, P. 'Reflections on the process of change on acute psychiatric wards during the City Nurse Project.' Journal of Psychiatric and Mental Health Nursing. 2006; 13(3): $260-268$. PMid:16737492 http://dx.doi.org/10.1111/j.1365-2850.2006.00932.x 